\title{
El rol de la asociatividad en la denominación de origen del bocadillo veleño colombiano como estrategia de competitividad
}

The role of the associativity in the designation of origin of the colombian bocadillo veleño as a competitive strategy

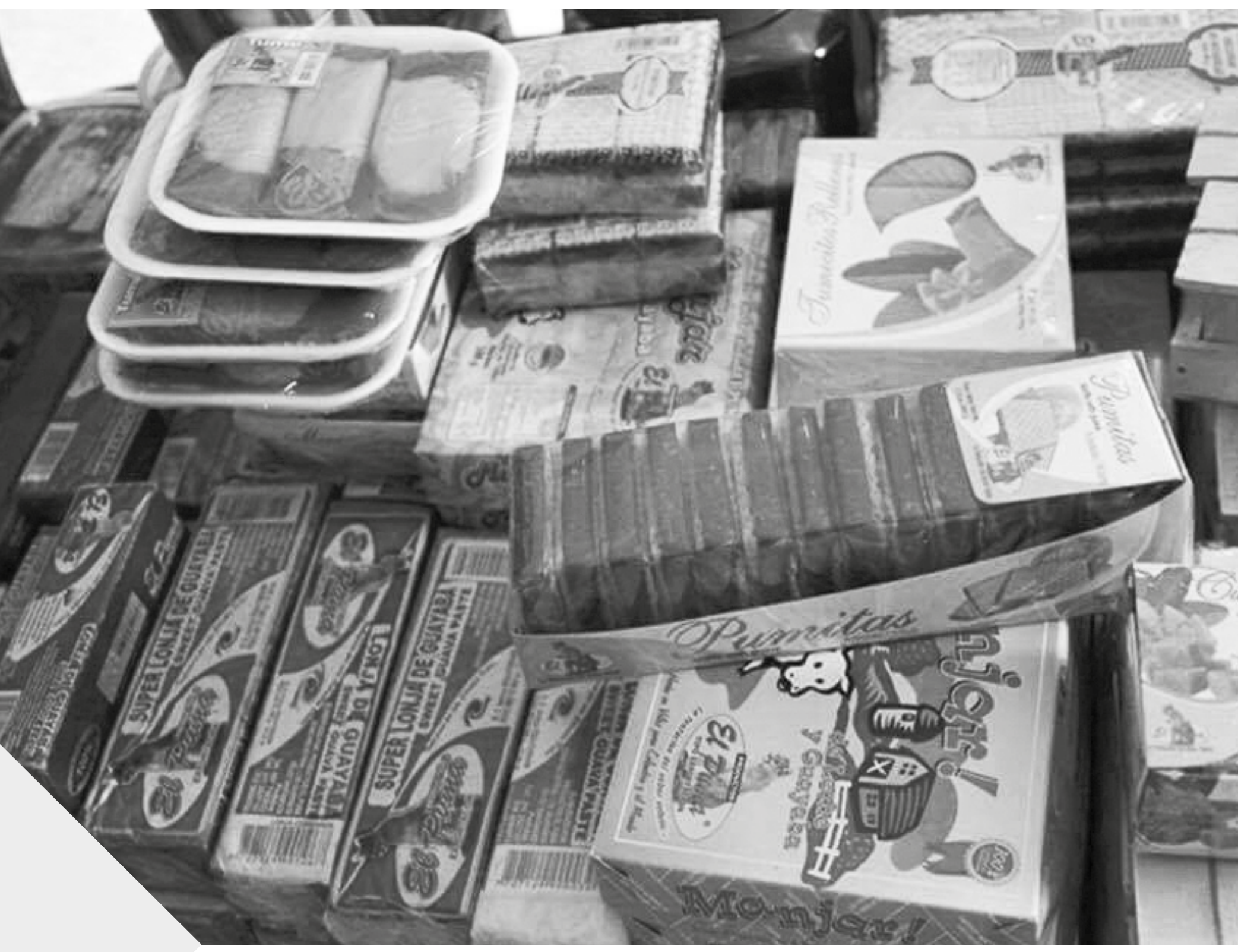




\title{
El rol de la asociatividad en la denominación de origen del bocadillo veleño colombiano como estrategia de competitividad $^{1}$
}

\section{The role of the associativity in the designation of origin of the colombian bocadillo veleño as a competitive strategy}

\author{
Andrea Johanna Muñoz Suárez², Yessica Yuliana Peña Castellanos³, Amanda Vargas Prieto ${ }^{4}$ \\ 'Universidad de La Salle, Bogotá, Colombia
}

Artículo recibido en agosto de 2016; artículo aceptado en octubre de 2016

Citación del artículo: Muñoz, A., Peña, Y. \& Vargas, A. (2017). El rol de la asociatividad en la denominación de origen del bocadillo veleño colombiano como estrategia de competitividad. I+D Revista de Investigaciones, 9(1), 27-37.

\begin{abstract}
Resumen
Esta investigación analiza las ventajas competitivas de diferenciación que podrían obtener las fábricas ubicadas en la Provincia de Vélez y Ricaurte con la obtención de la denominación de origen para el bocadillo veleño empacado en hoja de bijao y embalado en caja de madera. Por medio de una comparación entre el queso Paipa y el bocadillo veleño se demuestra la capacidad que tiene este último para generar procesos de desarrollo rural en la región. La comparación efectuada entre los dos productos permitirá analizar el contexto de los dos casos estudiados y evidenciar el rol de la asociatividad en el desarrollo local de los dos territorios (Provincia de Vélez y Paipa). Finalmente, se identifica que en el caso del queso Paipa la creación de procesos de asociatividad fue necesaria para obtener la denominación de origen, a diferencia del caso del bocadillo veleño, en donde la asociatividad facilita la obtención de dicha protección jurídica.
\end{abstract}

Palabras clave: Asociatividad, cadena de suministro, apelación, ventajas competitivas.

\begin{abstract}
This research analyzes the competitive advantages of differentiation that could get the factories located in the Province of Vélez and Ricaurte with obtaining the origin designation for "Bocadillo Veleño en hoja de bijao". Through a comparison of "Queso Paipa" and "Bocadillo Veleño" the processes of rural development in the region is demonstrated. The comparison made between the two products allow analyzing the context of the two cases studied and highlight the role of associativity in local development of both territories (Province of Velez and Paipa). Finally, it identifies that in the case of "Queso Paipa" creating association processes were necessary to obtain the origin designation, in the case of "Bocadillo veleño", the existence of the associativity facilitates obtaining such legal protection.
\end{abstract}

Keywords: Associativity, supply chain, appellation, competitive advantages.

1. Artículo de investigación, enfoque cualitativo, resultado de un proyecto de investigación culminado, perteneciente al área de Ciencias Económicas y Sociales, subárea de Finanzas y Comercio Internacional, desarrollado en el grupo de investigación Gidep de la Universidad de La Salle. Dirección: Carrera. 5 n. ${ }^{\circ}$ 59a-44, PBX: 3488000. Fecha de inicio: enero de 2015, fecha de terminación: abril de 2016.

2. Profesional en Finanzas y Comercio Internacional, Universidad de La Salle de la ciudad de Bogotá. Dirección: Carrera 5 Este n. $55-44$, Bogotá, Cundinamarca. Teléfono: (1) 3488000. Correo institucional: amunoz62@unisalle.edu.co.

3. Profesional en Finanzas y Comercio Internacional, Universidad de La Salle de la ciudad de Bogotá (Colombia). Dirección: Carrera 5 Este n. ${ }^{\circ} 55-44$, Bogotá, Cundinamarca. Teléfono: (1) 3488000. Correo institucional: ypena63@unisalle.edu.co.

4. Doctora en Ciencias Económicas, magister en Inteligencia Económica, administradora de empresas. Profesora asociada de la Universidad de La Salle Facultad de Ciencias Económicas y Sociales. Dirección: Carrera 5 Este n. ${ }^{\circ}$ 55-44, Bogotá, Cundinamarca. Teléfono: (1) 3488000. Correo institucional: amvargas@unisalle.edu.co 
La globalización ha impulsado una serie de elementos innovadores entre los que se encuentran tecnologías de avanzada, las cuales se caracterizan por crear nuevos patrones de consumo y una mayor conciencia sobre la preservación de los recursos naturales. Partiendo de ello, surge una reconceptualización de competitividad, donde las ventajas comparativas -motores de desarrolloevolucionan hacia las ventajas competitivas creadas a partir de la diferenciación del producto y de la disminución de costos.

Deestamanera, las nuevas estrategiasestán determinadas por el uso del conocimiento y la propiedad intelectual como vías de desarrollo para mejorar la competitividad. En este sentido, los activos intangibles se han convertido en fuente de valor para la empresa debido a que la propiedad industrial tiene como fin obtener ventajas sostenibles en el tiempo. De otra parte, ha establecido que uno de los signos distintivos más reconocidos es la denominación de origen, que permite proteger y distinguir los productos de la competencia, involucrando el saber hacer de los productores y la reputación que tiene el producto en una región determinada.

De acuerdo con lo anterior, es importante tener en cuenta la denominación de origen como estrategia de diferenciación, debido a que contribuye a identificar el valor de las características particulares de un producto, asociadas a su origen y a unos productores específicos. La asociatividad es un concepto que se ha estudiado en la literatura con la aparición de diferentes estrategias colectivas con el fin de responder a los desafíos de la globalización. Así, se encuentran autores como Porter (1991)ysudefinición declusters, Becattini (1962)y Marshall (1920) con la identificación de distritos industriales y Vásquez Barquero (1998) con su estudio sobre sistemas productivos locales. Dentro de esta perspectiva, en Colombia la Superintendencia de Industria y Comercio da este reconocimiento a los productores, fabricantes o artesanos autorizados para tener establecimientos en la región asignada. De esta manera, se entiende la asociatividad como "un mecanismo de cooperación empresarial en el que cada una de las empresas que participan, mantienen su independencia jurídica y su autonomía gerencial, decidiendo voluntariamente su participación en un esfuerzo conjunto con los otros participantes para la búsqueda de un objetivo común" (Rosales, 1997).

En la actualidad, existen veintidós productos con denominación de origen, once de ellos, artesanales; los productos más destacados son el café de Colombia representado por la Federación Nacional de Cafeteros, el queso Paipa con Asoquesopaipa, las achiras del Huila con su Asociación Bizcochosachira y el sombrero aguadeño, representado por la Asociación de Artesanos de Tuchín.

La provincia de Vélez y Ricaurte se ha destacado por su larga tradición en la elaboración del bocadillo veleño de guayaba y su respectiva cadena de suministro, en la cual se han determinado potencialidades productivas organizativas e institucionales. A partir de ello, los productores de la región tomaron la iniciativa de hacer uso de una herramienta de diferenciación competitiva para brindarle valor agregado al bocadillo veleño envuelto en hoja de bijao, por medio de la denominación de origen. Es por ello que esta investigación busca responder a la siguiente pregunta: ¿cómo la asociatividad contribuye a mejorar la competitividad de la cadena de valor y en especial el adecuado proceso de implementación de una denominación de origen en el caso del bocadillo veleño envuelto en hoja de bijao?

\section{Marco teórico}

\section{Teoría de la competitividad}

El punto de partida para abordar el concepto de competitividad es que el precio ya no es el único determinante de ventaja entre un agente económico y otro, sino que, por el contrario, existen muchos factores que determinan la manera de crear ventajas competitivas por medio de estrategias empleadas por los empresarios hasta los gobiernos, los cuales pueden hacer frente a la competencia y de esta manera generar crecimiento a nivel de la empresa, la industria y la nación. Inicialmente, Porter (1991) indica que la productividad es el determinante más influyente en la competitividad de una nación, de ahí la importancia de las empresas como creadoras de dicha productividad. En apoyo a esto, es fundamental mencionar que en el caso del bocadillo veleño, más que productividad, se necesita establecer una estrategia competitiva que permita a las empresas productoras lograr un empoderamiento en el mercado. Por esta razón, se debe tener en cuenta que la ventaja competitiva busca un enfoque favorable dentro de una industria, con el fin de establecer una posición rentable y sustentable frente a la competencia (Manrique, 2013).

A partir de esta premisa surgen nuevos paradigmas acerca de la competitividad tratados por nuevos autores del pensamiento económico, entre ellos lo expuesto por Pérez (1992), quien afirma que la competitividad es el resultado de la contribución de muchos niveles interrelacionados, desde el nivel de la empresa individual hasta el entorno regional. La empresa que obtiene éxito en los mercados internacionales debe preocuparse por la importancia de generar una red de interacciones 
nacionales para aumentar su calidad. En este sentido, el autor sostiene que la apertura económica de los mercados puede ser vista como un periodo de transición que lleva consigo un cambio de paradigmas para los empresarios.

Visto de otro modo, la competitividad no solo es la capacidad de transformar insumos en bienes y servicios para lograr una máxima utilidad, sino que integra las habilidades y condiciones requeridas para el ejercicio de la competencia, lo que quiere decir que se genera una ventaja sobre sus similares incorporando factores que proporcionan un desempeño superior al de la competencia. Sin embargo, no se puede hablar de la competitividad de un país, porque quienes compiten son esencialmente las empresas y los sectores económicos, es decir, que los actores de la competitividad internacional se reducen a unidades y sectores productivos.

Según Romo y Musik (2005), en los niveles concéntricos jerárquicos de competitividad -la empresa, la industria, la región y el país- los factores internos y externos señalan que la empresa es el centro de los niveles de competitividad. En el caso de la industria las variables más importantes son la diferenciación de productos, política del sector y los precios internacionales de los bienes producidos. Lo regional, por su parte, hace referencia a variables tales como la infraestructura requerida o la aglomeración, teniendo en cuenta la cantidad de plantas productoras en un mismo lugar. Finalmente, en términos del país, sus variables más destacadas son el comportamiento y volatilidad de las tasas de interés y de tipo de cambio.

En este sentido, la competitividad es trabajar conjuntamente, es decir implementar acciones colectivas que favorezcan a todos, y de este modo alcanzar mayores rendimientos; esto permite entender el papel que juega la asociatividad en la competitividad.

Entre los autores que han desarrollado este concepto se encuentra Vázquez (1998), quien afirma que un sistema productivo local se constituye a partir de varias empresas que se encuentran ubicadas en un mismo territorio y se especializan en la fabricación de un producto; dada esta condición, las empresas locales pueden alcanzar ventajas competitivas en mercados nacionales e internacionales. Por su parte, Porter (2003) define textualmente clúster como "concentraciones geográficas de empresas interconectadas, suministradores especializados, proveedores de servicios, empresas de sectores afines e instituciones conexas que compiten pero que también cooperan; y que pueden ser de carácter urbano, regional, nacional o supranacional" (p. 214). En consecuencia, debe entenderse que la base del desarrollo local es la asociatividad.

Para Alburquerque (2004) el desarrollo económico local destaca los valores territoriales, de identidad, diversidad y flexibilidad en cuanto a las formas de producción basadas en la industria y las características generales y locales de un territorio determinado. Así pues, puede afirmarse que en Colombia, en el departamento de Santander, existe una región que ha impulsado el desarrollo económico local por medio de elaboración del bocadillo veleño, la Provincia de Vélez y Ricaurte. Actualmente existen ciento treinta y una fábricas, ubicadas principalmente en los municipios de Vélez, Guavatá, Barbosa y Moniquirá, que hacen parte de esta cadena productiva, que han empezado a preocuparse por generar nuevas estrategias competitivas que permitan lograr un crecimiento de la industria y la región.

La literatura examinada, por su parte, brinda una amplia conceptualización de la competitividad identificando los factores más relevantes. Las teorías de Michael Porter, orientadas a la creación de importantes estrategias mundiales que impulsan el crecimiento de un sector económico, son la base principal de la competitividad. Porter (1991) afirma que en el escenario de la competencia moderna internacional, las empresas no necesitan quedarse en su país de origen, dado que pueden competir con estrategias mundiales en las que las actividades tienen lugar en muchos países. Pese a esto, se debe tener en cuenta el comportamiento del sector para tener la capacidad de ser estratégicamente diferenciado y de este modo hacer que la ventaja competitiva cree, primordialmente, valor para sus clientes.

Por esta razón, se puede decir que la clave de éxito de un sector u organización depende de la planeación por medio de la estrategia. A propósito de ello, Ohmae (1983) aborda la definición de estrategia como un plan de acción que consiste en lograr un desempeño superior en relación con la competencia y sus factores claves de éxito, combinando los puntos más fuertes de la empresa o del producto, teniendo en cuenta el desarrollo del ambiente competitivo. Porter (2002) considera que para llegar a ser estratégicamente diferenciado es importante implementar tres estrategias genéricas que son el liderazgo en costos, la diferenciación y el enfoque en la estrategia, que pueden ser utilizadas individualmente o en conjunto para crear una posición defendible que sobrepasa el desempeño de los competidores en una industria en el largo plazo.

Una de las estrategias más sobresalientes utilizada por los sectores económicos es la diferenciación, la cual 
establece que cada industria tiene sus propios medios para distinguirse, y por lo tanto, la lógica de dicha estrategia exige que una empresa elija atributos distintos a los de sus competidores, para lograr así sustentar que el precio especial supere los costos adicionales en que incurre su singularidad, demostrando que el precio elevado es realmente único.

En apoyo a lo anterior, Caldentey y Gómez (1996) sostienen que para los productores la diferenciación da lugar a una competencia monopolística que les permite incrementar sus precios; por el contrario, para los consumidores la diferenciación está asociada a una mayor satisfacción y a la capacidad de adaptarse a productos de diferentes segmentos de mercado. En el caso del bocadillo veleño la estrategia de diferenciación se asume como punto de partida o herramienta para distinguir el producto de su competencia nacional, y de esta manera, aprovechar las características especiales del producto, demostrando que existe una distinción entre el bocadillo de origen veleño y el bocadillo de otros departamentos.

De otra parte, es pertinente identificar el grado de asociatividad que se ha logrado en la Provincia de Vélez y Ricaurte, y que actúa como motor para alcanzar el desarrollo de la región.

\section{Activos intangibles, propiedad intelectual $y$ denominación de origen}

Después de haber indagado en las teorías de la ventaja competitiva, se evidencia que hoy en día se requieren nuevas estrategias para ser más competitivos, una de ellas es el conocimiento o capital intelectual, es decir, que las organizaciones deben hacer uso de sus conocimientos para incrementar su competitividad. Es por ello que existen activos intangibles que pueden ser utilizados en las organizaciones para lograr ventajas competitivas sostenibles en el tiempo, como la propiedad industrial. Uno de los signos distintivos de la propiedad intelectual es la denominación de origen, entendida como una estrategia de diferenciación que permite distinguir dicho producto o servicio de la competencia.

En este sentido, la economía del conocimiento hace referencia a la importancia que han tomado los activos intangibles como fuente de valor para la empresa. Al respecto, Kaplan y Norton (2000) argumentan que los activos intangibles dan la posibilidad de crear relaciones a largo plazo entre organizaciones y clientes, lo que contribuye a generar lealtad a medida que se ofrecen servicios específicos o se satisfacen demandas que antes no habían sido cubiertas, implementando altos estándares de calidad. Este conjunto de activos se clasifican en cuatro categorías según Brooking (1996): activos de mercado, propiedad intelectual, activos humanos y activos de infraestructura. De acuerdo con esto, se podría afirmar que la propiedad intelectual es una herramienta que permite proteger las expresiones culturales de una comunidad por medio de marcas colectivas como la denominación de origen, la cual no solo crea valor social si no que impulsa la asociatividad y el bien común debido a que está fundamentada en el saber hacer de los productores, la buena reputación y el reconocimiento que tiene el producto de una región determinada.

Teniendo en cuenta la relevancia de los activos intangibles en el mercado, cabe destacar que el municipio de Vélez está ligado a la elaboración del bocadillo veleño, que toma su nombre del gentilicio de esta región. Desde luego, quienes cuentan con el saber hacer del bocadillo son las personas que se dedican a su producción y están involucradas en la cadena de valor, es por ello que es necesario proteger la autenticidad del producto por medio del Proyecto Colipri, el cual hace referencia a la consecución de la denominación de origen específicamente para el bocadillo veleño envuelto en hoja de bijao y embalado en caja de madera.

La denominación de origen es un vínculo entre el producto y el espacio geográfico donde se elabora. Por supuesto, existe una sinergia entre el productor, su conocimiento y sus características autóctonas. Dicho vínculo nace como respuesta a la necesidad de reconocer y dar exclusividad a productos fabricados con cualidades típicas de una región su objetivo principal es proteger el producto de imitaciones ante industrias alternas.

En este sentido, la denominación de origen ha sido una de las características más significativas de la cultura europea y la reglamentación técnica - legal proveniente de Europa. Díaz (2011) plantea que durante mucho tiempo productos como la champaña, el vino de Jerez, el vino de Oporto o el coñac, se elaboraban en diferentes lugares y los obstáculos para comercializarlos eran escasos. Sin embargo, finalizando el siglo XIX, los productores de Inglaterra protestaron y exigieron una diferenciación por su procedencia, de esta forma nace el "Made in..." o "Hecho en..." conocido como el auténtico antecedente de la protección de los productos caracterizados por su calidad y sistemas de elaboración. No obstante, se evidenciaban constantes inconformidades en cuanto a la procedencia de los productos, por ello, en 1891 se firmó el Arreglo de Madrid donde se estableció que la diferencia de calidad en los productos era muy importante, pero lo era aún más en productos alimenticios. Es por esto que 
se inicia un proceso internacional para tratar el tema. En efecto, hubo un lapso considerable para que hubiese acuerdos internacionales positivos, como lo fueron las Convenciones de Stresa el 1 de junio de 1951 y de la Haya el 18 de julio de 1951.

De este modo, los artesanos de productos alimenticios tradicionales identificaron la necesidad de individualizar sus productos con marcas creadas a partir de los nombres geográficos del lugar de fabricación, lo que permite que las denominaciones de origen sean protegidas en diferentes países del mundo en donde el marco de la protección, el grado y la especificidad varían según el caso. Cabe destacar que según Espejel, Fandos y Flavian (2007) las denominaciones de origen son importantes en tanto actúan como indicadores de calidad del comportamiento del consumidor. En el caso, por ejemplo, del aceite de oliva del Bajo Aragón, los autores señalan la influencia de la calidad, la lealtad y la intención de compra que ofrece un consumidor cuando adquiere un producto alimenticio con denominación de origen, con el fin de conocer la importancia que los consumidores brindan a exigencias como las materias primas, territorio, y el origen.

En Colombia, son veintidós productos tradicionales los que han obtenido la denominación de origen, el más significativo es el café de Colombia que goza de protección jurídica con el fin de incentivar a pequeños productores agrícolas a enfrentar las dificultades inherentes del mercado internacional de bienes por medio de la incursión en nuevos segmentos en la cadena de valor. Lozano, Samper y García (2011) sostienen que dicha connotación es una barrera de entrada ante competidores que buscan imitar el producto o aprovecharse indebidamente de su reputación, lo que genera un poder de negociación por parte de los productores. Además, uno de los beneficios derivados de la denominación de origen del café de Colombia fue conquistar la lealtad de los consumidores.

Ahora bien, con los ejemplos citados se evidencia que la denominación de origen es utilizada para productos que provienen principalmente de un lugar determinado y que poseen características derivadas de la localización, entre ellas las condiciones ambientales, la tradición y la habilidad o conocimiento propio de los productores. De esta forma, la denominación de origen determina la relación que hay entre el producto y su lugar geográfico. Por su parte, las indicaciones geográficas y las denominaciones de origen son vistas como régimen contractual de los beneficiarios y de las ventajas que trae consigo el sometimiento a un régimen contractual organizado; adicionalmente, estas comprenden los ámbitos de aplicación y las diferencias que tiene la denominación de origen con otros derechos de propiedad industrial. En apoyo a esta afirmación, la investigación de Vázquez y Valverde (2011) indica que un producto que está protegido por una identificación geográfica o una denominación de origen posee ventajas comparativas y competitivas en relación con un producto genérico. En último lugar se infiere que la denominación de origen se le otorgará al bocadillo veleño envuelto en hoja de bijao debido a que la Superintendencia de Industria y Comercio consideró que el producto era apto para a acogerse a dicho signo distintivo. Por lo tanto, al obtener la denominación de origen, tanto las fábricas como la región se verán favorecidas para adquirir ventajas competitivas tales como factores de diferenciación ante productos genéricos, reconocimiento del producto según su región geográfica y vínculo entre las características, la calidad y la procedencia del producto.

Para ello, es importante identificar el rol de la asociatividad en la denominación de origen del bocadillo veleño envuelto en hoja de bijao y embalado en caja de madera como estrategia de competitividad para alcanzar el desarrollo local en la Provincia de Vélez y Ricaurte, debido a que este proceso debe estar motivado por la acción y el liderazgo de los actores empresariales locales y regionales, organizados en asociaciones, sin dejar de lado la identidad de los campesinos y el proceso de toma de decisiones.

\section{El rol de la asociatividad en la denominación de origen como estrategia de competitividad}

Las nuevas tendencias de mercado exigen a las organizaciones ser competitivas nacional e internacionalmente. En este sentido, es necesario considerar la teoría de la ventaja comparativa de David Ricardo y la ventaja competitiva de Michael Porter, indicando que la diferenciación se lleva a cabo cuando una ventaja comparativa se convierte, por medio de la estrategia, en una ventaja competitiva. En este sentido, Porter, por medio de su teoría, se refiere al valor agregado que recibe un bien o un servicio cuando alcanza una posición preferencial en el mercado y logra que las empresas y el sector económico sean ubicados en un punto de vista diferenciador ante sus consumidores, generando una fidelización determinante en el momento de efectuar la compra.

La diferenciación de un producto puede adquirirse a través de las características esenciales que contemplan las necesidades del consumidor; los activos innovadores, como son los intangibles, abarcan la propiedad 
intelectual e industrial como herramienta que permite reconocer quién lo hizo o de donde viene; además, uno de sus factores más sobresalientes es el sostenimiento en el largo plazo. Ahora bien, desde la perspectiva del consumidor se determina que este mide el valor de un producto por los factores o atributos extras que le ofrece dicho producto, mientras que las empresas buscan añadir a sus productos rasgos o cualidades que los diferencien de otros. La aparición de las marcas ha sido uno de los hechos más significativos, llegando a convertirse en uno de los principales instrumentos competitivos frente a las grandes industrias. Los consumidores son los principales dadores de valor a las marcas por su influencia en el momento de compra, puesto que se enfrentan a una decisión que abarca la utilidad que le genera con base en cualidades, precio y satisfacción. Según algunos estudios sobre la reacción entre la percepción de calidad y las marcas, el consumidor prefiere las marcas nacionales, es decir que esto podría ser un beneficio para la denominación de origen.

En este caso, la denominación de origen es una marca nacional que distingue productos con ciertas características especiales, por esta razón, se asume que los consumidores perciben estos productos con la palabra distintiva como de mayor calidad. Las marcas colectivas son signos que permiten identificar el origen geográfico, la manera en que es fabricado un producto y sobre todo, son un medio para proteger la cultura y las cualidades propias de un territorio. Por ende, la denominación de origen obtiene una reputación colectiva, dando lugar a un monopolio colectivo, en donde los beneficiarios son directamente los productores de una región determinada.

Según Enrique (2002), la asociatividad es una estrategia orientada a potenciar el logro de una ventaja competitiva por medio de la cooperación o el establecimiento de nuevos acuerdos con otras empresas para efectuar ciertas actividades dentro de la cadena de producción; dicha estrategia conduce a un posicionamiento de la empresa en un mercado nacional e internacional. Este proceso de asociatividad da lugar a un incremento de la producción y la productividad junto con la participación y autonomía de las empresas asociadas, generando un mayor poder de negociación y mejora en la calidad de los productos. Es por ello que la denominación de origen requiere indiscutiblemente de la asociatividad, es decir, que quienes tomen la iniciativa sean los productores y agentes de la cadena productiva que se encuentran organizados en asociaciones, con el fin de proteger la identidad cultural de su comunidad.
En este sentido, y en el contexto de este estudio, se destaca el importante papel de Asoveleños, Guajaba y Asobijao en la provincia de Vélez y Ricaurte para la consolidación de la denominación de origen del bocadillo veleño envuelto en hoja de bijao embalado en caja de madera, como una estrategia de competitividad que permitirá diferenciar el producto y fortalecer tanto a los productores como a la industria de la región, incrementando su productividad y generando un reconocimiento de las características autóctonas que brinda al bocadillo la Provincia de Vélez y Ricaurte.

\section{Método}

\section{Tipo de estudio}

Esta investigación se realiza de acuerdoa una metodología mixta. Según Hernández, Collado y Baptista (2008), dicha metodología comprende un conjunto de procesos sistemáticos, empíricos y críticos de investigación que implican la recolección y el análisis de datos cualitativos y cuantitativos, así como su integración para lograr un mejor entendimiento del estudio a realizar. De este modo, el enfoque cualitativo proporciona a profundidad los datos, detalles y experiencias únicas, riqueza interpretativa, contextualización del ambiente o entorno, y un punto de vista fresco y natural. Debido a que el tipo de estudio a tratar es exploratorio - formulativo, la naturaleza de este indica que se toma de referencia el enfoque cualitativo y a partir de sus resultados se trata un enfoque cuantitativo, el cual ofrece la posibilidad de generalizar los resultados, otorga control sobre los fenómenos, brinda un punto de vista de conteo, permite conocer un enfoque sobre puntos específicos y facilita la comparación sobre estudios similares.

Para esta investigación, el enfoque cualitativo está encaminado a una caracterización del sector confitero y del municipio de Vélez a través de la recolección de información en fuentes primarias y secundarias. Además, por medio de la entrevista semiestructurada se hace una contextualización de Asoveleños, donde se identifican los puntos fuertes y las condiciones del Proyecto Colipri; asimismo, se hace una visita de campo a una de las fábricas para conocer las condiciones de producción del bocadillo veleño. Luego, se realiza una revisión bibliográfica sobre el queso Paipa como producto nacional con denominación de origen e igualmente se realiza una visita de campo y una entrevista semiestructurada.

A partir de los resultados obtenidos se implementa el enfoque cuantitativo por medio de una comparación del bocadillo veleño con el queso Paipa; para ello, se 
identifican indicadores en cuanto a la especificidad de los recursos territoriales y la importancia socioeconómica cultural del producto, se elaboran cuadros comparativos para determinar las ventajas competitivas que adquiriría bocadillo veleño envuelto en hoja de bijao al recibir la denominación de origen, y los beneficios para los productores de la región que estén asociados. Finalmente, a partir de los resultados obtenidos, se podrán establecer las conclusiones sobre las ventajas de competitividad - diferenciación para el bocadillo veleño envuelto en hoja de bijao y el rol de la asociatividad en este proceso.
Para poner en práctica el modelo de Aranda, Gómez y Ramos (2014) se acudió a documentos, trabajos de investigación, visita de campo a las fábricas del queso Paipa y del bocadillo veleño; además, se realizaron entrevistas semiestructuradas dirigidas a productores, líderes de las asociaciones y funcionarios de las fábricas con presencia en los territorios de Paipa y la Provincia de Vélez. El cuestionario de la entrevista se estructuró con preguntas abiertas que podían dar respuesta a cada uno de los indicadores del modelo a tratar; las visitas de campo se realizaron durante el primer semestre del año 2015.

Tabla 1

Indicadores de la especificidad de recursos territoriales e Importancia socioeconómica y cultural del producto

\begin{tabular}{|c|c|c|c|c|c|c|c|}
\hline \multirow{2}{*}{ Indicador } & \multicolumn{3}{|c|}{ Criterios y escala de calificación } & \multirow{2}{*}{ Indicador } & \multicolumn{3}{|c|}{ Criterios y escala de calificación } \\
\hline & Baja & Media & Alta & & Baja & Media & Alta \\
\hline $\begin{array}{c}\text { Procedencia de la materia } \\
\text { prima principal }\end{array}$ & $\begin{array}{c}\text { Variedades o razas } \\
\text { introducidas }<25 \\
\text { años }\end{array}$ & $\begin{array}{c}\text { Variedades o razas } \\
\text { adaptadas }>25 \\
\text { años }\end{array}$ & $\begin{array}{l}\text { Variedades o } \\
\text { razas endémicas }\end{array}$ & $\begin{array}{l}\text { Participación de la } \\
\text { producción y área } \\
\text { agrícola del territorio }\end{array}$ & $\begin{array}{c}\text { Menos de } 20 \% \\
\text { del área agrícola } \\
\text { sembrada }\end{array}$ & $\begin{array}{c}\text { Entre } 20 \% \text { y } 50 \% \\
\text { del área agrícola } \\
\text { sembrada }\end{array}$ & $\begin{array}{c}\text { Más de } 50 \% \text { del } \\
\text { área agrícola } \\
\text { sembrada }\end{array}$ \\
\hline $\begin{array}{l}\text { Incidencia de las } \\
\text { condiciones } \\
\text { edafoclimáticas }\end{array}$ & $\begin{array}{l}\text { Irrelevante. No } \\
\text { influye en la } \\
\text { calidad del } \\
\text { producto }\end{array}$ & $\begin{array}{l}\text { Puede influir. Sin } \\
\text { Ilegar a ser una } \\
\text { condición }\end{array}$ & Determinante & $\begin{array}{c}\text { Participación de } \\
\text { generación de empleo en } \\
\text { el territorio }\end{array}$ & $\begin{array}{l}\text { Menos de } 5 \% \text { de } \\
\text { los empleados } \\
\text { del territorio }\end{array}$ & $\begin{array}{c}\text { Entre el } 5 \% \text { y el } \\
20 \% \text { de los } \\
\text { empleados del } \\
\text { territorio }\end{array}$ & $\begin{array}{c}\text { Más del } 20 \% \text { de los } \\
\text { empleados del } \\
\text { territorio }\end{array}$ \\
\hline $\begin{array}{l}\text { Origen del saber hacer en el } \\
\text { proceso productivo }\end{array}$ & $\begin{array}{l}\text { Inducido menos } \\
\text { de } 25 \text { años }\end{array}$ & $\begin{array}{c}\text { Inducido hace más } \\
\text { de } 25 \text { y meno de } 80 \\
\text { años }\end{array}$ & $\begin{array}{l}\text { Saber hacer } \\
\text { autóctono del } \\
\text { territorio }\end{array}$ & $\begin{array}{l}\text { Estabilidad de } \\
\text { organizaciones }\end{array}$ & $\begin{array}{c}\text { Mas } 50 \% \text { existe } \\
\text { hace más de un } \\
\text { año }\end{array}$ & $\begin{array}{l}\text { Más del } 50 \% \text { existe } \\
\text { entre } 1 \text { y } 5 \text { años }\end{array}$ & $\begin{array}{c}\text { Más del } 50 \% \\
\text { existen hace más } \\
\text { de } 5 \text { años }\end{array}$ \\
\hline Especialidad del trabajo & $\begin{array}{l}\text { No existe, o puede } \\
\text { ser adquirido por } \\
\text { calificación } \\
\text { externa }\end{array}$ & $\begin{array}{l}\text { Sí, por un vínculo } \\
\text { con la actividad } \\
\text { productiva } \\
\text { calificación local }\end{array}$ & $\begin{array}{c}\text { Sí, prima el } \\
\text { conocimiento } \\
\text { adquirido por } \\
\text { legado }\end{array}$ & $\begin{array}{l}\text { Tiempo de arraigo del } \\
\text { saber hacer del territorio }\end{array}$ & Menos a 25 años & Entre 25 y 50 años & Mayor a 50 años \\
\hline $\begin{array}{l}\text { Conocimiento específico } \\
\text { relacionado con la } \\
\text { producción }\end{array}$ & No existe & $\begin{array}{c}\text { Sí existe, } \\
\text { generalizado entre } \\
\text { la población local }\end{array}$ & $\begin{array}{c}\text { Sí existe } \\
\text { conocimiento } \\
\text { particular de } \\
\text { algunos actores }\end{array}$ & $\begin{array}{c}\text { Actividades culturales } \\
\text { relacionadas al producto } \\
\text { y al territorio }\end{array}$ & No existen & $\begin{array}{l}\text { Por lo menos una } \\
\text { actividad de } \\
\text { notoriedad local }\end{array}$ & $\begin{array}{c}\text { Por lo menos una } \\
\text { festividad de } \\
\text { notoriedad } \\
\text { nacional } \\
\end{array}$ \\
\hline
\end{tabular}

Fuente: Tipificación de los vínculos productos típicos - territorio: una metodología con aplicación empírica en productos agroalimentarios de la región andina de Colombia (2014).

\section{Resultados}

El resultado final se determina a partir del modelo de Aranda, Camacho y Ramos (2014) en donde se proponen cuatro escenarios de acuerdo con el promedio de las escalas de cada uno de los indicadores de la especificidad de recursos territoriales y la importancia socioeconómica y cultural del producto: el primero está formado por los productos típicos y sectores específicos, que constituyen la base de un sector especializado localizado en el territorio, con poca participación de la producción en la actividad económica de este; el segundo, por los productos típicos y distritos industriales, entre los que se destaca la industrialización endógena o espontánea, caracterizada por el liderazgo del empresario local y la creación de instituciones; el tercero, está conformado por los productos típicos y por las zonas marginales en las cuales, a pesar de que la producción en otras regiones sea de mejor calidad y más competitivas, se mantiene la actividad económica en el territorio; y la cuarta, por la valorización autóctona de productos genéricos cuya producción se puede realizar en diferentes regiones, si bien, debido a sus características, los consumidores tienen cierta preferencia por el producto de un determinado territorio.

\section{Productos típicos y sectores específicos - queso Paipa}

El queso Paipa, como producto con denominación de origen, se ubica en el escenario de productos típicos y sectores específicos, ya que la región de Paipa y Sotaquirá es un territorio que se ha especializado en su elaboración, pero aun así ni su producción ni los actores vinculados a esta tienen una participación significativa 
Tabla 2

Tipificación de los vínculos producto típico - territorio

\begin{tabular}{cccc}
\hline & & \multicolumn{2}{c}{ Importancia socioeconómica y cultural de producto } \\
\cline { 2 - 4 } & & Baja & Alta \\
\hline \multirow{2}{*}{$\begin{array}{c}\text { Especificidad de } \\
\text { los recursos } \\
\text { territoriales }\end{array}$} & Alta & $\begin{array}{c}\text { Productos típicos y sectores } \\
\text { específicos }\end{array}$ & $\begin{array}{c}\text { Producto típicos y distritos } \\
\text { industriales }\end{array}$ \\
\cline { 2 - 4 } & Queso Paipa & $\begin{array}{c}\text { Bocadillo veleño envuelto en hoja de } \\
\text { bijao }\end{array}$ \\
& Baja & Productos típicos y zonas & Valorización autóctona de los \\
& & marginales & productos genéricos \\
\hline
\end{tabular}

Fuente: Tipificación de los vínculos productos típicos - territorio: una metodología con aplicación empírica en productos agroalimentarios de la región andina de Colombia (2014).

en la actividad económica derivada de dicho queso. Esto es notorio en la poca cantidad de personas empleadas en la cadena productiva, el bajo nivel de asociatividad y la falta de empoderamiento en la realización de actividades locales alusivas a su producto autóctono. De esta manera, se evidencia que Asoquesopaipa fue creada después de obtener la denominación de origen, lo cual explica que haya poca estabilidad en las organizaciones y que sus productores todavía no tengan la confianza para hacer parte de alguna de ellas. Sin embargo, se pudo establecer que para la formalización de la denominación de origen los productores deben acogerse a alguna asociación, gremio, organización o club que les permita beneficiarse de las ventajas competitivas que brinda dicho signo distintivo.

\section{Productos típicos y distritos industriales - bocadillo veleño envuelto en hoja de bijao}

Como candidato a recibir la denominación de origen, el bocadillo veleño está ubicado en productos típicos y distritos industriales debido a que obtuvo una calificación promedio alta para ambos indicadores, hecho que puede considerarse como un escenario ideal para que productos tradicionales puedan obtener mayores ventajas competitivas, gracias a la incidencia en los mercados nacionales.

Este escenario se caracteriza por aprovechar las capacidades internas de una región con el fin de lograr una economía sustentable y sostenible en el tiempo, en donde la actividad económica es una agroindustria propia de la región de la que se derivan otras industrias relacionadas con el producto. Un ejemplo de esto es que en la cadena productiva del bocadillo se desencadenan pequeñas industrias tales como la recolección y preparación de la hoja de bijao y la fabricación de la caja de madera, ambas directamente relacionas con la presentación del producto su distribución. Además, los productos típicos y distritos industriales se caracterizan por el liderazgo del empresariado local y la presencia de instituciones de apoyo, lo cual se ve reflejado en el nivel de asociatividad de la provincia de Vélez y Ricaurte, en donde existe una asociación por cada eslabón de la cadena de valor, la cual permite regular y facilitar la coordinación entre los actores que la conforman; estas son Asoveleños, Guajaba, Asobijao, Asofrutas y Cooperativas Guayabas Veleñas.

Un aspecto a destacar del liderazgo de los empresarios en la región es que la proactividad de cada gremio los ha llevado a pensar en los requisitos necesarios para obtener la denominación de origen, para lo cual están proyectando en crear una federación (Fedeveleños) en representación de todas las asociaciones existentes en la región involucradas en la actividad económica del bocadillo. En cuanto al consejo regulador, requisito fundamental de la denominación de origen, Fedeveleños será quien constatará y supervisará el manual regulatorio y la junta directiva será la encargada de expedir el manual operativo y administrativo de la denominación de origen. Asimismo, una de las iniciativas de Asoveleños, incluida en el plan de desarrollo de la región es la construcción de una planta para consolidar el proceso productivo, y lograr que sea uniforme, teniendo en cuenta el perfil exportador. Algunos de los valores agregados de Asoveleños son la experiencia, su conocimiento del mercado, el poder de negociación que demostraron al comprar un terreno que era necesario para el desarrollo de su actividad la realización del estudio de los tipos de ingenierías, la gestión que adelantan para recaudar los recursos y adquirir apoyo de entes como la gobernación y el Ministerio de Industria Comercio y Turismo. 
Finalmente, al tratar el queso Paipa como producto con denominación de origen y al bocadillo veleño envuelto en hoja de bijao como candidato a recibir esta distinción, se encuentra que el bocadillo veleño presenta características que pueden convertirse en herramientas de competitividad que le permitirían alcanzar una diferenciación del producto y un reconocimiento, no solo a nivel nacional sino internacional, tal como lo expone Porter (2002). En cuanto al queso Paipa, se observa que no hay un aprovechamiento total de las ventajas competitivas que puede brindarle la denominación de origen, debido a la falta de estabilidad y organización en las asociaciones, asimismo, a la ausencia de información en los requerimientos que se deben tener en cuenta para formalizar este proceso.

\section{Conclusión}

Aunque el bocadillo veleño envuelto en hoja de bijao es producto autóctono de características especiales por su origen, no ha tenido una acogida en el mercado internacional; por ello, se espera que con esta herramienta de competitividad puedan implementarse algunas acciones que permitan que el producto impulse el crecimiento y desarrollo de la región. También se espera que se convierta en un rubro representativo para el sector de chocolatería, confitería y materias primas, toda vez que la denominación de origen y las exigencias internacionales -las normas técnicas y sanitariaspueden ayudarlo a obtener un buen desempeño en el comercio internacional. La actividad comercial del bocadillo veleño está orientada a países como Estados Unidos y España; de otra parte, los productores de la región -por medio de Asoveleños y Guajaba- se han encargado de introducir el producto en el país.

Lo expuesto permite afirmar que la denominación de origen es una estrategia de diferenciación competitiva, que a través de procesos asociativos beneficia a los productores de una región determinada, da a conocer el producto, impulsa el desarrollo regional y protege el nombre del producto ante sus competidores Romo y Musik (2005) .

Por otra parte, el modelo de Aranda, Gómez y Ramos (2014) propone indicadores que se ajustan perfectamente a los factores determinantes involucrados en la denominación de origen La relación entre el producto típico y el territorio, y la importancia que tiene la actividad económica en la región, permite afirmar que dichos indicadores fueron esenciales para identificar las ventajas que ha adquirido el queso Paipa con la denominación de origen, las cuales están relacionadas con la capacitación en la cadena de suministro, principalmente al primer, eslabón correspondiente a la materia prima principal, y reconocimiento del producto a nivel nacional, factores que en conjunto han impulsado el desarrollo de bocadillo en la región.

En síntesis, se encontró que en el caso del queso Paipa primero se adquirió la denominación de origen, y luego, para mantener dicha denominación, se crearon programas de asociatividad en la región. Por el contrario, en el caso del bocadillo veleño, y pese a estar en proceso para la obtención de denominación de origen -retomando la teoría de Alburquerque (2004) sobre el desarrollo económico local-se ha encontrado que hay un alto grado de asociatividad en toda la cadena productiva involucrada en el bocadillo. Además, se observa que la denominación de origen realmente puede convertirse en una herramienta competitiva de diferenciación debido a que el bocadillo veleño tradicional envuelto en hoja de bijao y empacado en caja de madera es un producto que la mayoría de fábricas ubicadas en la provincia de Vélez y Ricaurte producen, por ende las fabricas se verán beneficiadas, puesto que el objetivo principal del proyecto Colipri es proteger el nombre de este producto ante empresarios de otras regiones.

De otra parte, durante las visitas de campo se identificó la presencia de información asimétrica debido a que no todos los productores de la región tienen conocimiento de los requerimientos de la denominación de origen. Para que todos los productores logren obtener la denominación de origen deben hacer parte de alguna asociación, organización o grupo que les permita beneficiarse de las ventajas competitivas que brinda la denominación de origen.

\section{Referencias}

Alburquerque, F. (2004). Sistemas productivos locales: una mirada desde la política económica local para la generación de empleo. Seminario Cepal. Buenos Aires.

Aranda, Y., Gómez, A. \& Ramos, E. (2014). Tipificación de los vínculos productos típicos - territorio: una metodología con aplicación empírica en productos agroalimentarios de la región andina de Colombia. Agroalimentaria, 20(38).

Becattini, G. (1962). Il concetto di industria e la teoría del valore. Turín: Boringhieri.

Brooking, A. (1996). Intellectual Capital, Asset for the Third Millenium Enterprise. International Thomson Business Press. London. 
Caldentey, P. \& Gómez, A. (1996). Productos típicos territorio y competitividad. Agricultura y sociedad.

Díaz, I. (2011). Denominaciones de origen e indicaciones geográficas como garantía de calidad. España.

Enrique, C. (2002). El Salvador: la asociatividad empresarial entre pymes. San Salvador: Iberpyme. Recuperado de http:// www.iberpymeonline.org/noticias. asp?step $=1$ \&id $=147$.

Espejel, J., Fandos, C. \& Flavian, C. (2007). La importancia de las denominaciones de origen protegidas para indicadores de calidad para el comportamiento del consumidor. El caso del aceite de oliva del Bajo Aragón. Zaragoza: Universidad de Zaragoza.

Hernández, R., Collado, C. \& Baptista, P. (2008). Metodología de la investigación (4a. ed.).

Kaplan, R. \& Norton, D. (2000). Cuadro de mando integral. Barcelona: Gestión 2000.

Lozano, A., Samper, L. \& García, J. (2011). Simposio mundial sobre indicaciones geográficas. Lima: Indecopi.

Manrique, O. (2013). Verdades en las iniciativas de clúster en el departamento de Santander. I+D Revista de Investigaciones, 1(2), 30 - 41.

Marshall, A. (1920). Principles of Economics. London: Macmillan.

Ohmae, K. (1983). La mente del estratega: el triunfo de los japoneses en el mundo de los negocios. Bogotá: Mc Graw Hill.
Pérez, C. (1992). La modernización industrial en América Latina y la herencia de la sustitución de importaciones. Ottawa.

Porter, M. (1991). La ventaja competitiva de las naciones (p. 163). Buenos Aires: Argentina S.A.

Porter, M. (2002). Ventaja Competitiva. México: Continental. Porter, M. (2003). Clusters, Convergence and Economic Performance. New York: Regional Studies.

Romo, D. \& Musik, G. (2005). Sobre el Concepto de Competitividad (pp. 202-201). Bogotá: Comercio Exterior.

Rosales, R. (1997). La Asociatividad como estrategia de fortalecimiento de las PyMes. México: Universidad de Texas Bueno.

Vázquez, A. (1998). Desarrollo endógeno. Conceptualización de la dinámica de las economías urbanas y regionales. Cuadernos del Cendes, (38).

Vázquez, C. \& Valverde, F. (2011). Las Indicaciones geográficas y las denominaciones de origen: Régimen contractual de los beneficiarios con una indicación geográfica o denominación de origen y las ventajas que trae consigo el sometimiento a un régimen contractual organizado. Santiago, Chile: Universidad de Chile. 